Supporting Information

\title{
Comparative analysis of the hydrogen bond vibrations of ice XII
}

Xiao-Qing Yuan, Xu-Hao Yu, Xu-Liang Zhu, Xue-Chun Wang, Xiao-Yan Liu, JingWen Cao, Xiao-Ling Qin, Peng Zhang*

Email: zhangpeng@sdu.edu.cn

School of Space Science and Physics, Shandong University, Weihai 264209, China. 


\section{Scheme S1:}

hbond.py

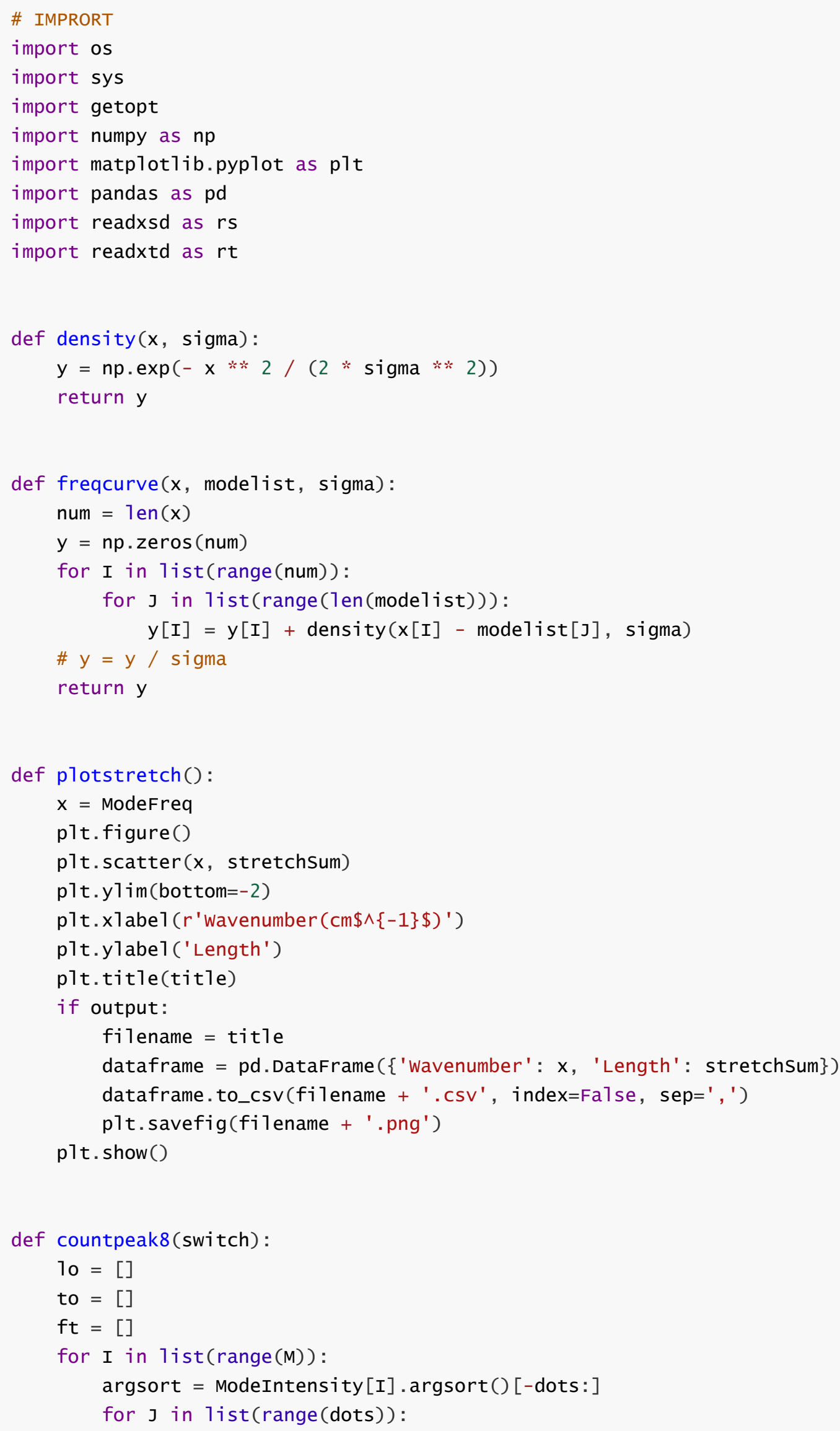


if intLim[0] $<=$ ModeIntensity[I][argsort[J]] $<=$ intLim[1]:

loAng $=\operatorname{modeBi}$ sAng $[\mathrm{I}][\operatorname{argsort}[\mathrm{J}]]$

toAng $=\operatorname{modeEdgeAng}[\mathrm{I}][\operatorname{argsort}[\mathrm{J}]]$

if switch:

toAng $=\operatorname{modeTOAng}[\mathrm{I}][\operatorname{argsort}[\mathrm{J}]]$

1imit $=($ loAng + toAng $) *$ angRatio

if $170<$ ModeFreq[I] < 180 :

$\mathrm{ft}$. append (ModeFreq $[\mathrm{I}]$ )

e1se:

if loang <= 1imit - criticang:

10. append (ModeFreq[I])

elif toAng $<=$ limit - criticang:

to. append (ModeFreq $[I]$ )

p1t.figure()

num $=1000$

$\mathrm{x}=\mathrm{np} .1$ inspace (ModeFreq.min() $-3 *$ smooth, 400, num)

$\# \mathrm{x}=$ np.7inspace (ModeFreq.min() $-3 *$ smooth, ModeFreq. $\max ()+3 *$ smooth, num)

$\mathrm{y} 1$ = freqcurve $(\mathrm{x}, 10$, smooth $)$

$y 2$ = freqcurve $(x$, to, smooth $)$

y3 = freqcurve $(x, f t$, smooth $)$

p1t.p1ot $(x, y 1, c=' b ')$

p1t.fi11_between $(x, 0, y 1$, color='b', alpha=0.3)

p1t.p1ot $\left(x, y 2, c=' r^{\prime}\right)$

p1t.fi11_between ( $x, 0, y 2$, color $=' r '$ ' a1pha $=0.3)$

p1t.plot $(x, y 3, c=' g r e e n ')$

p1t.fi11_between ( $x, 0, y 3$, color='green', a1pha=0.3)

\# 标记

p1t.x1im(freqLim[0]-3*4, freqLim[1] $+3 * 4)$

p1t.ylim(0, 6)

p1t.x1abe1 ( $r$ 'Wavenumber $\left.(\mathrm{cm} \$ \wedge\{-1\} \$)^{\prime}\right)$

plt.ylabel ('Counts')

\# plt.yticks([])

plt.title(title)

if output:

filename $=$ title $+{ }^{\prime}-m 2 d^{\prime}+{ }^{\prime}-c{ }^{\prime}+\operatorname{str}($ criticang $)+{ }^{\prime}-I\left[^{\prime}+\right.$ $\operatorname{str}(\operatorname{round}(\operatorname{intLim}[0], 3))+', ' \backslash$

$+\operatorname{str}(\operatorname{round}(\operatorname{intLim}[1], 3))+']-F\left[{ }^{\prime}+\operatorname{str}(\operatorname{round}(\right.$ freqLim[0], 3)) +

$+\operatorname{str}($ round (freqLim[1], 3)) + '] -s ' + str(smooth)

dataframe $=$ pd.DataFrame $(\{$ 'Wavenumber': $x$, 'TO': y1, 'LO': y2 $\})$

dataframe.to_csv(filename + '.csv', index=False, sep=', ')

plt.savefig(filename + '.png')

p1t. show()

def countpeak (switch):

$10=[]$

to $=[]$

$\mathrm{ft}=[]$

for I in 7 ist(range $(M))$ :

argsort $=$ ModeIntensity $[\mathrm{I}] \cdot \operatorname{argsort}()[-\operatorname{dots}:]$

for $\mathrm{J}$ in 1 ist(range(dots)):

if intLim[0] $<=\operatorname{ModeIntensity[I][argsort}[J]]<=\operatorname{intLim[1]:}$ 


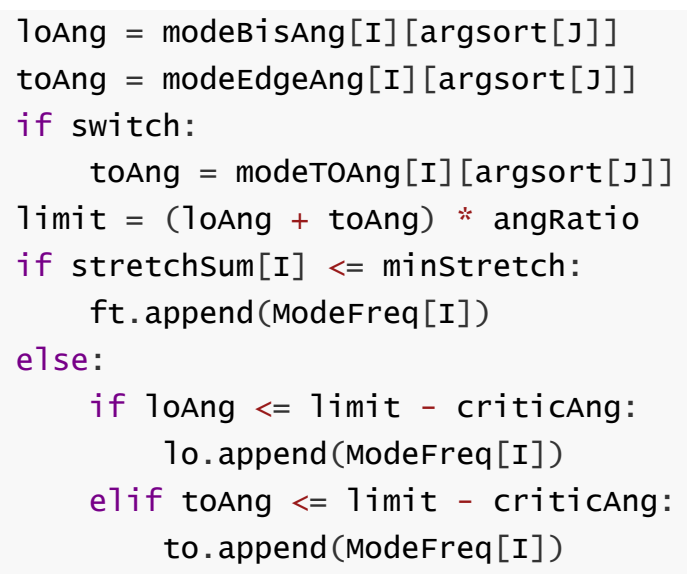

def usage(): 
print (u"'"

-h /--help : help

"" ")

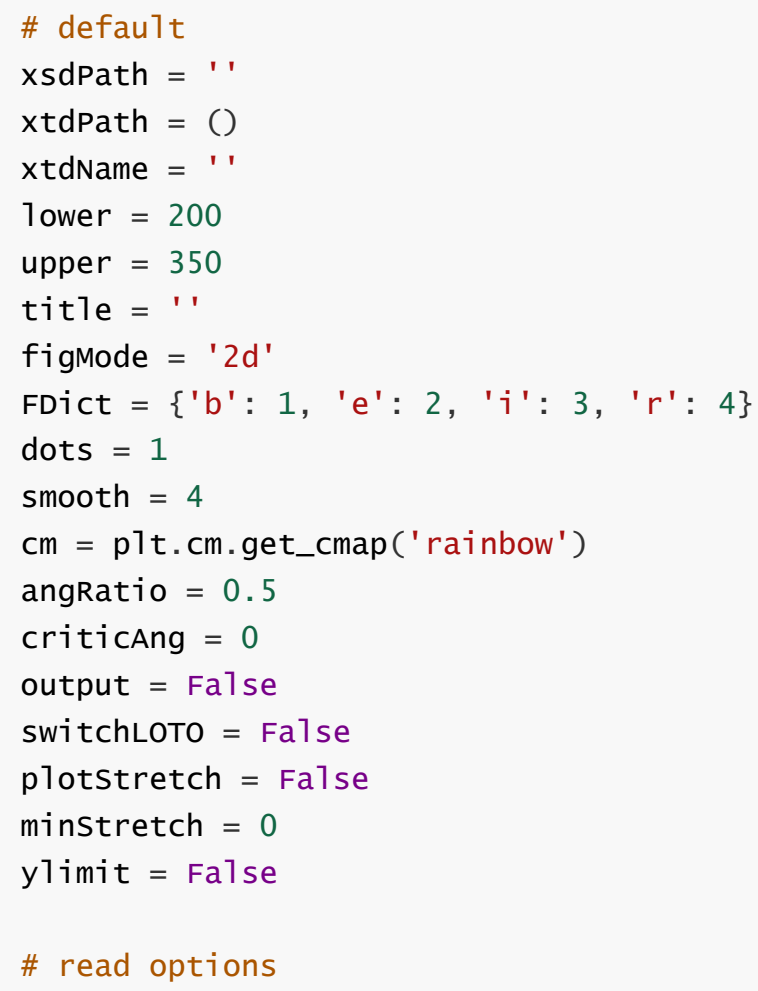




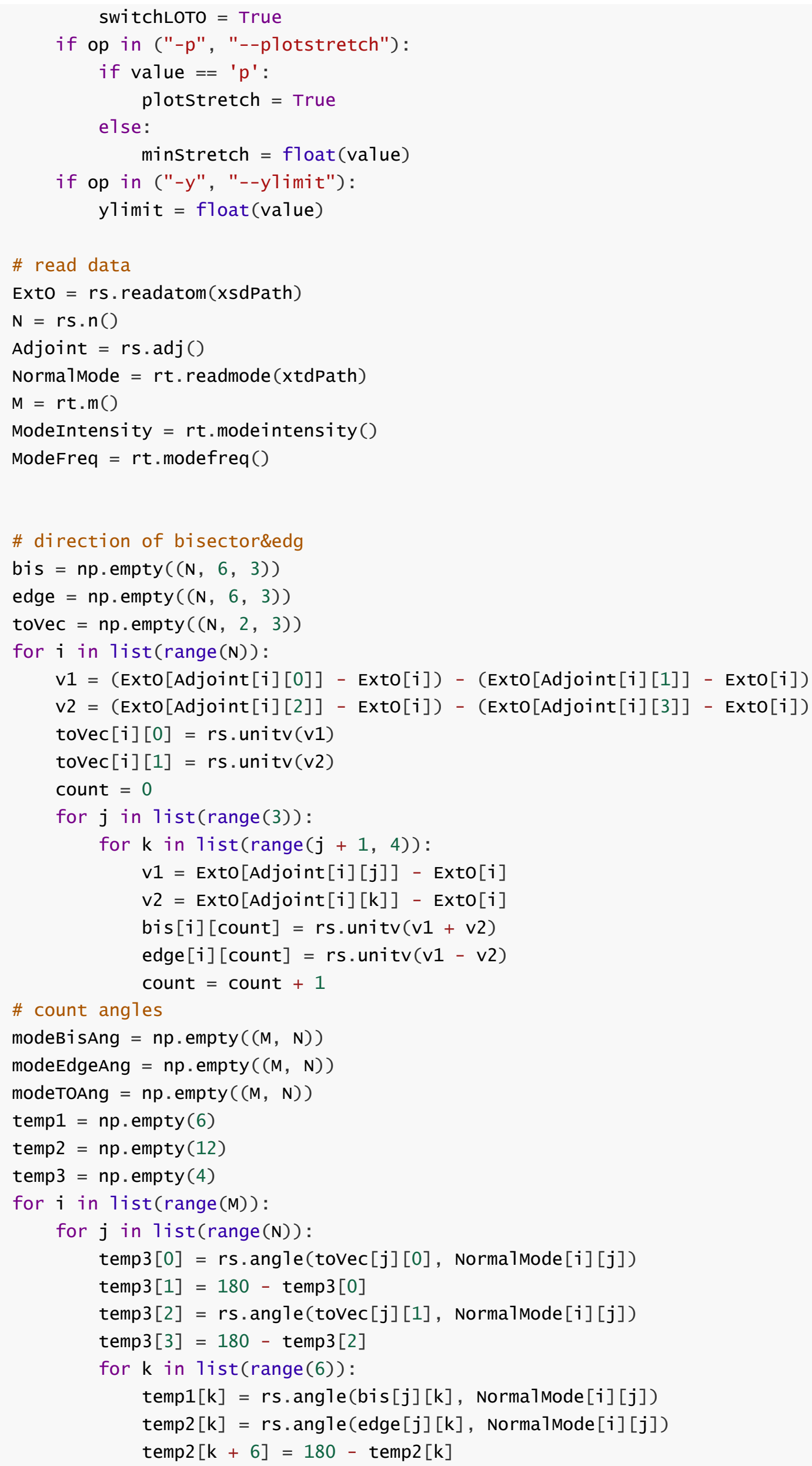




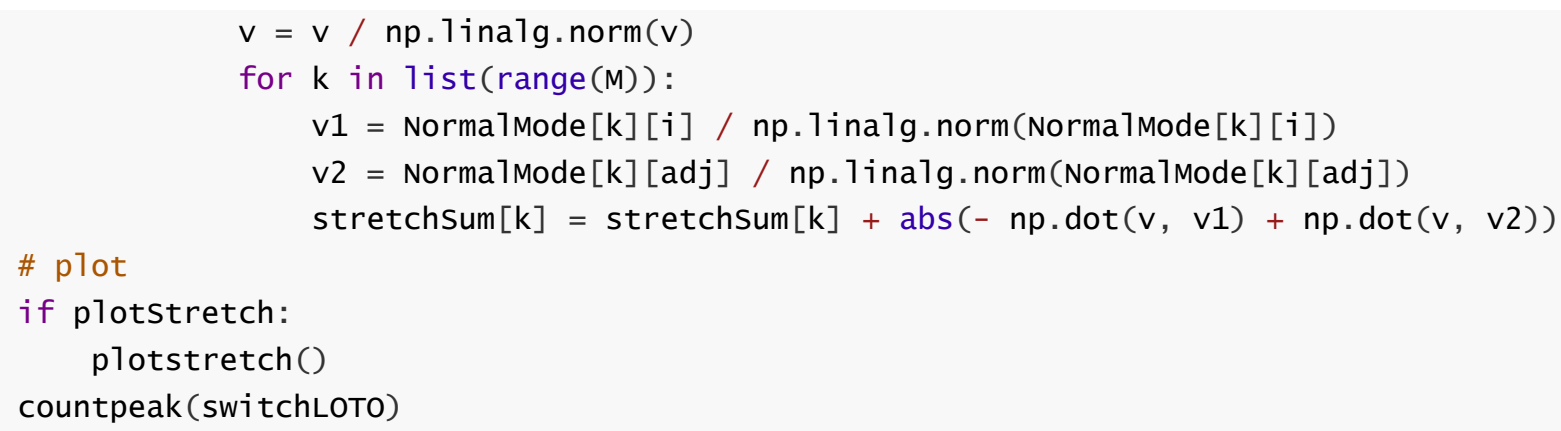

\section{Scheme S2:}

readxsd.py

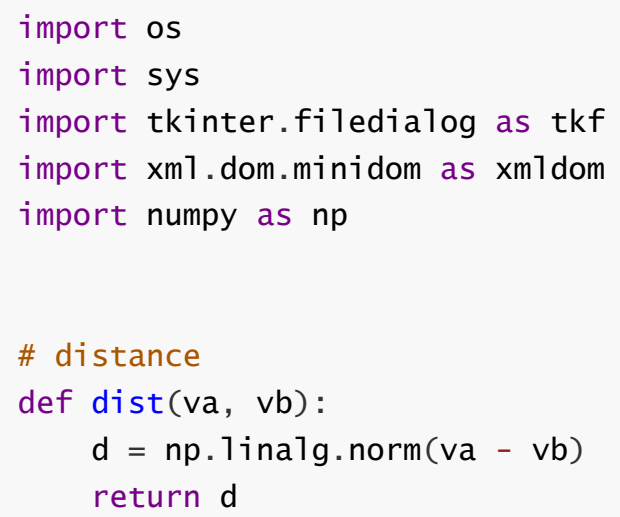


data $=$ 1ist $(\operatorname{map}($ float, stringdata.split(",")))

return data

def readatom(xsdPath):

\# Declare global variables

global Noxygen

global LattVector

global adjoint

\# File selection dialog

if xsdpath $==$ ' ' :

print("Please choose a structure file")

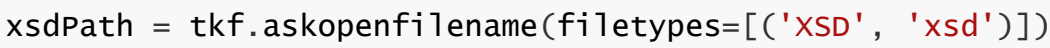

if $\mathrm{xsdPath}==$ ' ' :

print ('No chosen file')

sys.exit(0)

e1se:

print ('--xsdfile="' + xsdPath + '"')

\# Get file object

print("Read:", os.path.basename(xsdPath))

xsdFile $=x m 1$ dom. parse (xsdPath)

\# Get element object

StruElement $=$ xsdFile. documentElement

\# Get subtag

SpaceGroup = StruElement.getElementsByTagName ("SpaceGroup")

Atom3d = StruElement.getElementsByTagname ("Atom3d")

\# Get spaceGroup tag attribute:A, B, CVectors

AVector = np.array (str2data (SpaceGroup[0].getAttribute("AVector")))

BVector = np.array (str2data (SpaceGroup[0].getAttribute ("BVector")))

cVector $=$ np.array $($ str2data $($ SpaceGroup [0].getAttribute ("cvector")))

\# Get Atom3d tag attribute

\# atom amount

NHydorgen $=0 \quad \# \mathrm{H}$ atom amount

Noxygen $=0 \quad \#$ o atom amount

for $i$ in 1ist(range(1en(Atom3d))) :

if Atom3d[i].getAttribute("Components") == "H":

NHydorgen $=$ NHydorgen +1

elif Atom3d[i].getAttribute("Components") == "O":

Noxygen $=$ Noxygen +1

\# atom coord and ID (X,Y, Z, ID)

hFracXYZ = np.empty ((NHydorgen, 3))

oFracXYZ = np.empty $(($ NOxygen, 3$))$

countH $=0$

counto $=0$

for $i$ in 1 ist $(($ range $(1$ en (Atom3d)))):

if Atom3d[i].getAttribute("Components") == "H":

hFracXYZ[countH] = str2data(Atom3d[i].getAttribute("XYZ"))

countH = countH +1

elif Atom3d[i].getAttribute("Components") == "O":

oFracXYZ[counto] = str2data(Atom3d[i].getAttribute("XYZ"))

counto $=$ counto +1

LattVector $=$ np. concatenate $($ (AVector, BVector, $\operatorname{CVector})) \cdot \operatorname{reshape}(3,3)$

\# LattVector $=$ LattVector. $\mathrm{T}$ 


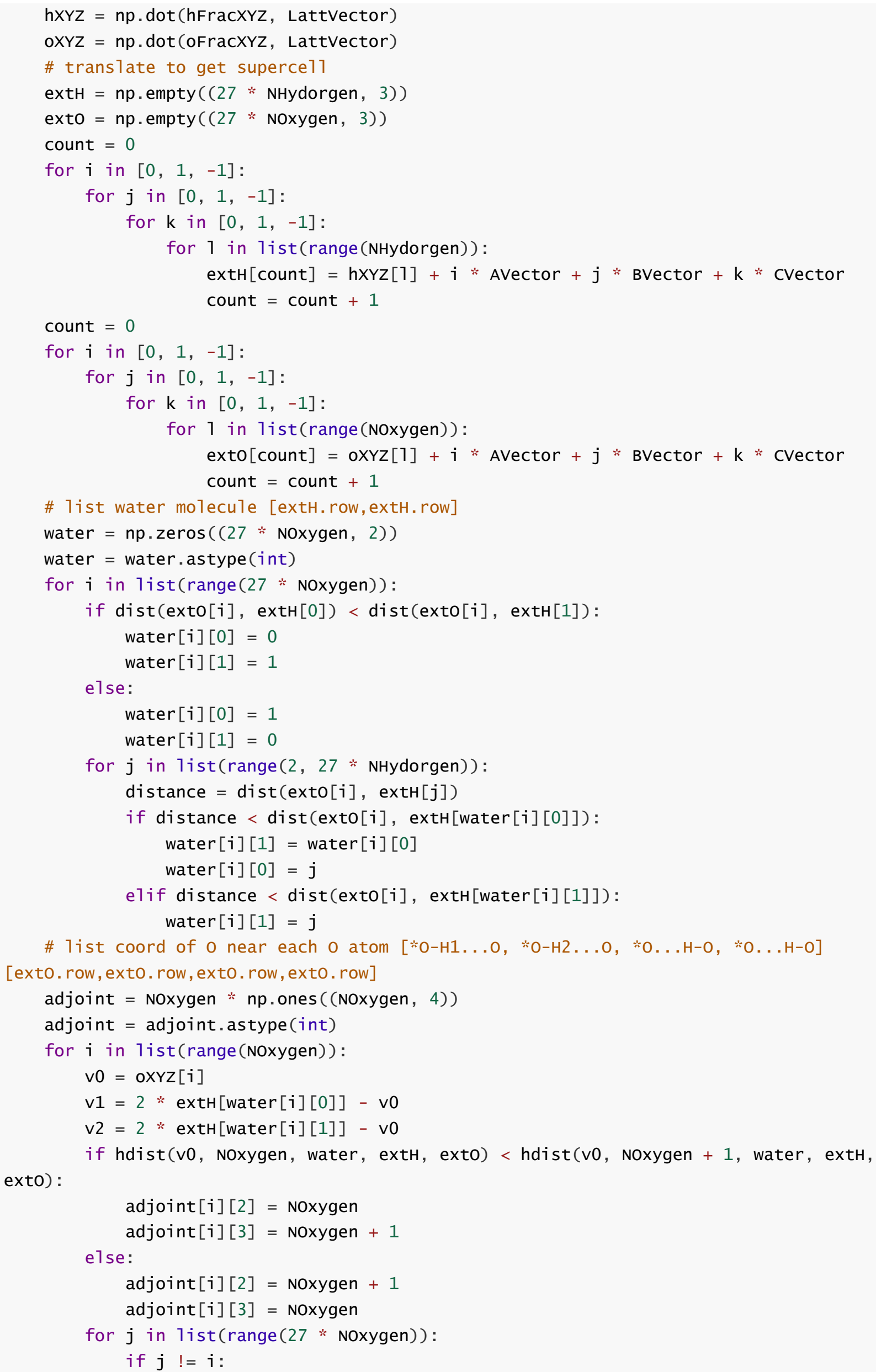




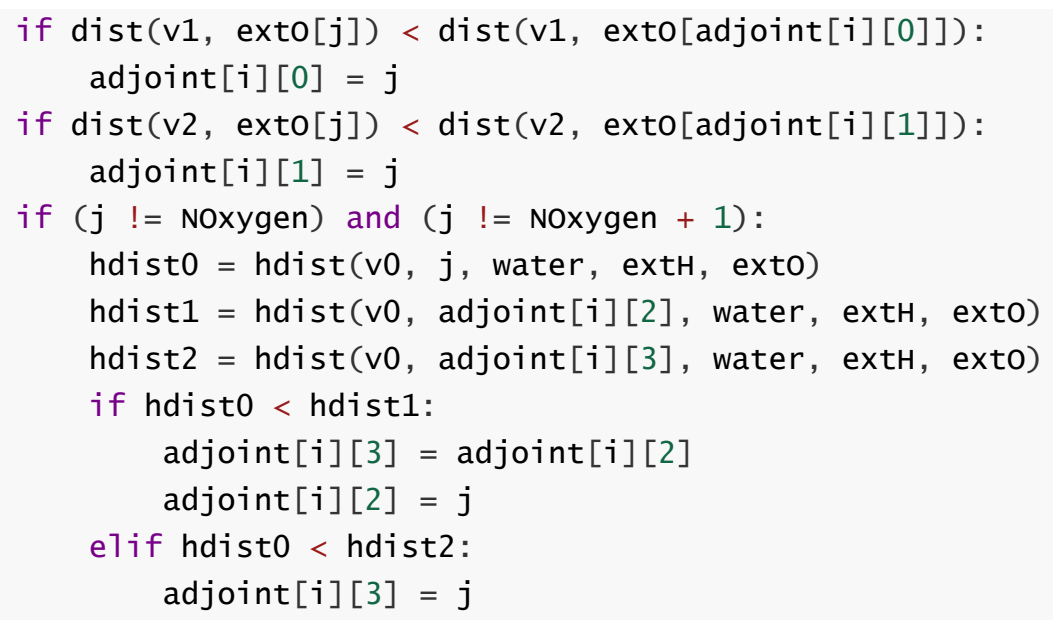

return exto

def $n()$ :

return Noxygen

def lattvector():

return Lattvector

def $\operatorname{adj}()$ :

return adjoint

\section{Scheme S3:}

readxtd.py

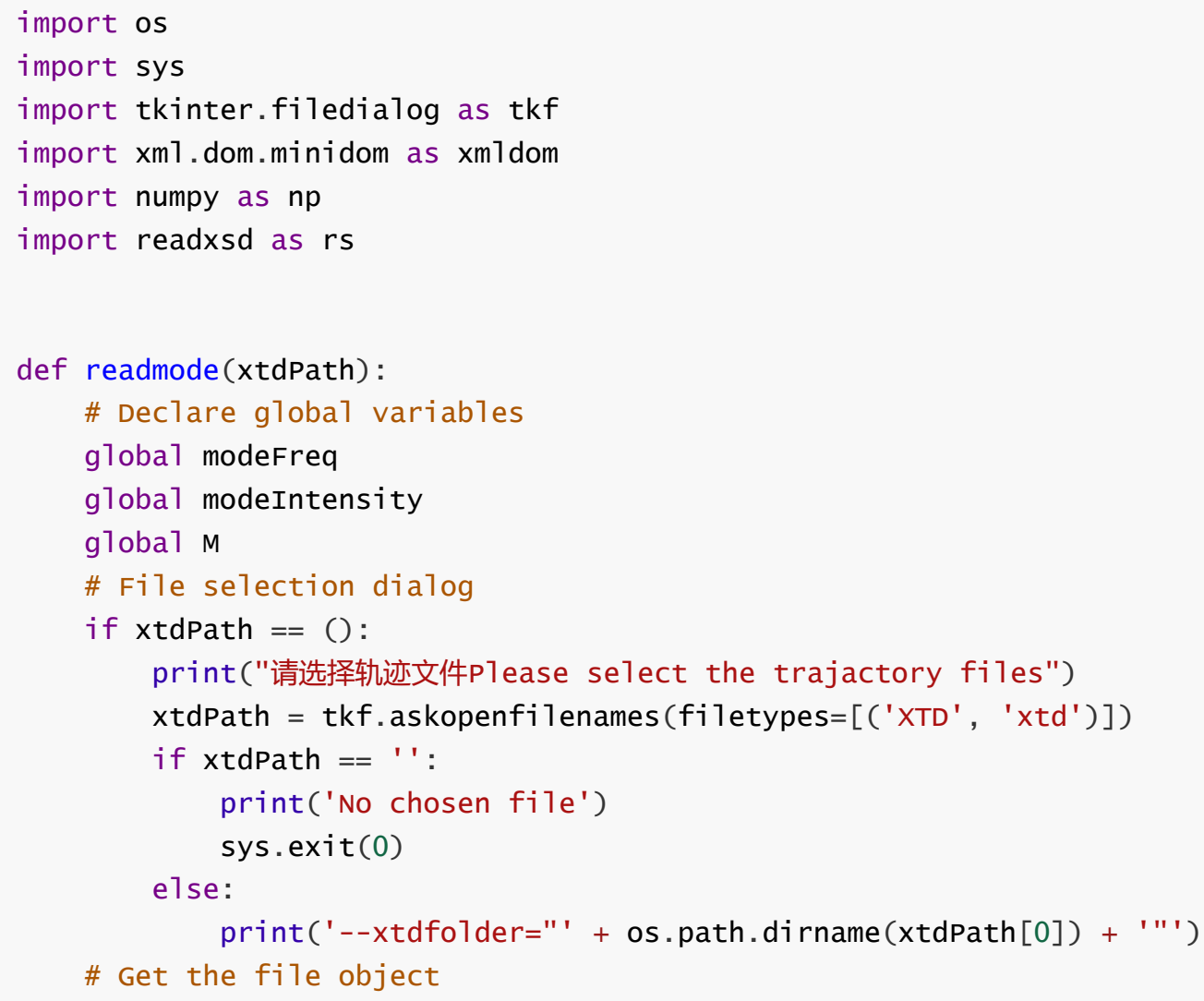




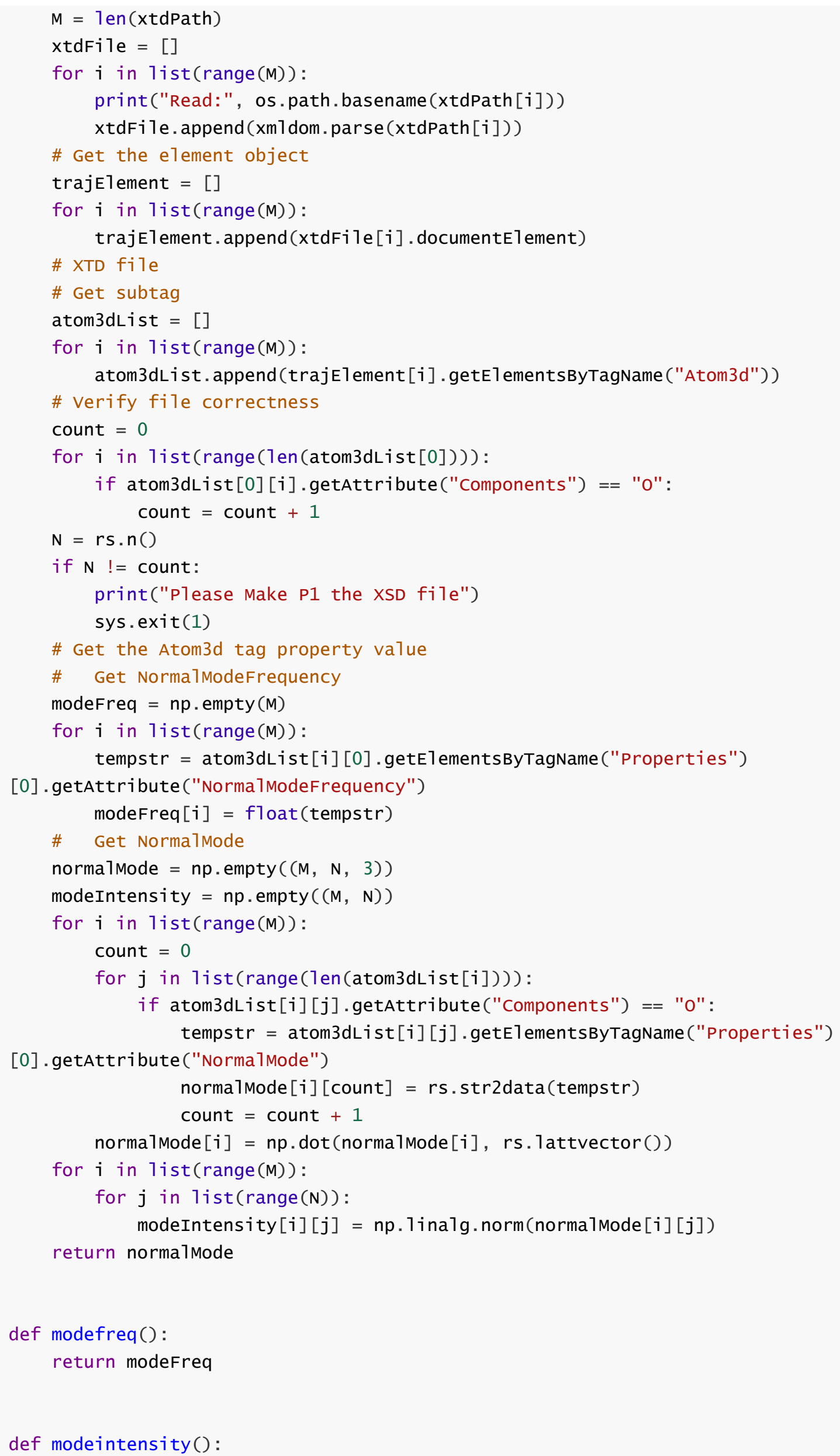


return modeIntensity

def $m()$ :

return M 